\title{
Canonical form of Hamiltonian matrices
}

\author{
A. P. Zuker, L. Waha Ndeuna, F. Nowacki, and E. Caurier, \\ IRES, Bât27, IN2P3-CNRS/Université Louis Pasteur BP 28, F-67037 Strasbourg Cedex 2, France
}

(July 9, 2018)

\begin{abstract}
On the basis of shell model simulations, it is conjectured that the Lanczos construction at fixed quantum numbers defines - within fluctuations and behaviour very near the origin - smooth canonical matrices whose forms depend on the rank of the Hamiltonian, dimensionality of the vector space, and second and third moments. A framework emerges that amounts to a general Anderson model capable of dealing with ground state properties and strength functions. The smooth forms imply binomial level densities. A simplified approach to canonical thermodynamics is proposed.
\end{abstract}

21.10.Ma,21.60.Cs,21.60.-n

Since many physical problems can be thought as matrix diagonalizations, much attention has been devoted to the general properties of matrices, starting with Wigner's Gaussian Orthogonal Ensemble (GOE) which has provided invaluable insight into fluctuation properties [1, 2, 2]. However, physical systems are usually associated to Hamiltonians of rank $(r) 1+2$ (i. e., 1+2-body) while for GOE the rank is the dimensionality of the space. Two Body Random Ensemble (TBRE) or Embedded GOE (EGOE) are the suggested denominations for limited ranks. We shall prefer to speak of Hamiltonian matrices, either random or not. Exact results and even numerical ones are hampered by the difficulty of enforcing the rank conditions, which are hard to simulate through special band or sparse matrices, and it would be desirable to have a canonical form that ensures rank $1+2$. It is our aim to show that the Lanczos tridiagonal construction can provide it. (The impatient reader may proceed to the discussion after Fig. 11)

The algorithm is standard [3]:

A starting vector (the pivot) $|0\rangle$ is chosen. The vector $\left|a_{0}\right\rangle=\mathcal{H}|0\rangle$ has necessarily the form $\left|a_{0}\right\rangle=H_{00}|0\rangle+$ $\left.\left|1^{\prime}\right\rangle\right\rangle$, with $\left\langle 0 \mid 1^{\prime}\right\rangle=0$. Calculate $\left\langle 0 \mid a_{0}\right\rangle=H_{00}$. Normalize $\left|1^{\prime}\right\rangle$, to find $H_{01}=\left\langle 1^{\prime} \mid 1^{\prime}\right\rangle^{1 / 2}$. Iterate until state $|k\rangle$ has been found. The vector $\left|a_{k}\right\rangle=\mathcal{H}|k\rangle$ has necessarily the form

$$
\left|a_{k}\right\rangle=H_{k k-1}|k-1\rangle+H_{k k}|k\rangle+\left|(k+1)^{\prime}\right\rangle,
$$

Calculate $\left\langle k \mid a_{k}\right\rangle=H_{k k}$. Extract and normalize $\left|(k+1)^{\prime}\right\rangle$, to find $H_{k k+1}=\left\langle(k+1)^{\prime} \mid(k+1)^{\prime}\right\rangle^{1 / 2}$.

The choice of pivot is arbitrary and it can be adapted to special problems. One of the most interesting is the calculation of strength functions: Given a transition operator $\mathcal{T}$, act with it on a target state $|t\rangle$ to define a pivot $|0\rangle=\mathcal{T}|t\rangle$ that exhausts the sum rule for $\mathcal{T}$. The tridiagonal matrix elements are then linear combinations of the moments of the strength distribution $\left\langle 0\left|\mathcal{H}^{K}\right| 0\right\rangle$ (expand $|0\rangle$ in eigenstates to check the statement). Therefore the eigensolutions of the $I \times I$ matrix define an approximate strength function $S_{I}(E)=\sum_{i=1, I} \delta\left(E-E_{i}\right)\langle i|\mathcal{T}| 0\rangle^{2}$, whose first $2 I-1$ moments are the exact ones (state $|I\rangle$ is obtained by orthogonalizing $\mathcal{H}^{I-1}|0\rangle$ to the preceeding states. Hence $H_{I I}=\left\langle 0\left|\mathcal{H}^{2 I-1}\right| 0\right\rangle+$ information from previous iterations) The eigenstates act as "doorways" whose strength will be split until they become exact solutions for $I$ large enough. To fix ideas consider in Fig. 1 the first 200 Lanczos matrix elements calculated to reproduce the Gamow Teller strength $(\mathcal{T}=\sigma \tau)$ obtained through the reaction ${ }^{48} \mathrm{Ca}(\mathrm{p}, \mathrm{n}){ }^{48} \mathrm{Sc}$. In Ref 700 iterations proved necessary to ensure the exact $S(E)$ in the $p f$ shell (the dimension of the $J T=13$ space is $d=8590$ ). The question at the origin of the present work is: do we truly need 700 exact eigensolutions to obtain a good $S(E)$ ? Probably not, and the idea is to calculate exactly a few matrix elements, and replace the rest by an adequate approximation.

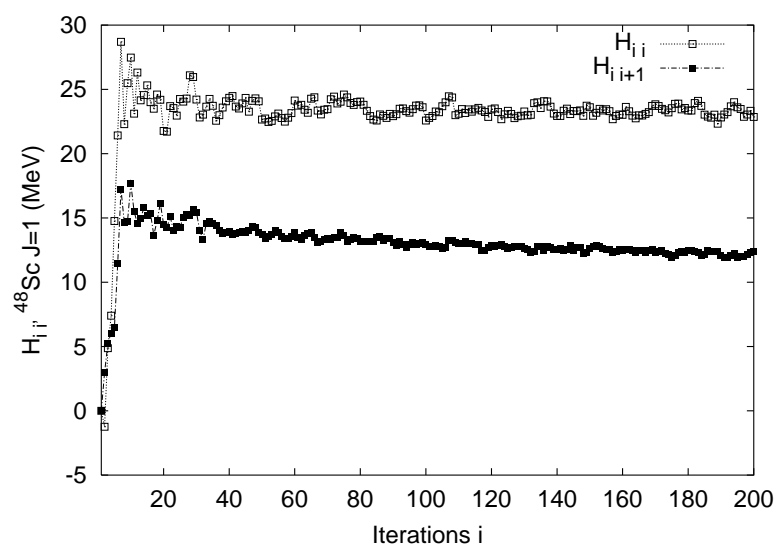

FIG. 1. Exact matrix elements for ${ }^{48} \mathrm{Sc}(J=1)$

The very rapid variation of the first matrix elements in Fig. 11 certainly depends on the pivot. It is followed by fluctuations around smooth patterns: a constant for the diagonals, and a line $b$ for the off diagonals. Therefore, a good approximation to the exact problem comes in three steps. First determine the smooth patterns, then do the few Lanczos iterations necessary to reach them and finally replace the rest of the matrix elements by fluctuations mounted on the smooth patterns. 
Next we concentrate on the first step.

The quantities that turn out to be necessary are the dimensionality, which we write as $d=2^{N}$, the centroid $E_{c}=d^{-1} \operatorname{tr}(H)$, the variance $\sigma^{2}$ and skewness coefficient $\gamma_{1}$ :

$$
\begin{aligned}
d & =2^{N} \Longrightarrow N=\ln d / \ln 2, \quad E_{c}=d^{-1} \operatorname{tr}(H) \\
\sigma^{2} & =d^{-1} \operatorname{tr}\left(H-E_{c}\right)^{2}, \gamma_{1}=\left(d \sigma^{3}\right)^{-1} \operatorname{tr}\left(H-E_{c}\right)^{3} .
\end{aligned}
$$

An inverse binomial function is also needed: $x=\operatorname{nib}(b, N), 0<x<1 / 2$, the inverse of

$$
b=\operatorname{bin}(x)=e^{\frac{1-N}{2}[(1+2 x) \ln (1+2 x)+(1-2 x) \ln (1-2 x)],}
$$

the Stirling approximation of

$$
b=\operatorname{bin}(x, N)=2^{-N}\left(\begin{array}{c}
N \\
N(1 / 2-x)
\end{array}\right),
$$

a binomial shifted and normalized so as to be maximum and unity at the origin.

The "data" from which we draw conclusions are exact diagonalizations (at fixed angular momentum $J$ ) for ${ }^{48} \mathrm{Ca}$, a typical closed shell nucleus [5], and for the configurations $\left(f_{7 / 2} p_{3 / 2}\right)_{\pi}^{4}\left(g_{9 / 2} d_{5 / 2}\right)_{\nu}^{4}\left((f p g d)^{8}\right.$ for short $)$ that exhibit rotational behaviour with backbend [6].

The matrix elements in Figs. 2 and 3 correspond to maximum $d$ for each family. They are totally representative of the other members. Only the general (pivotindependent) trend is visible.

To within fluctuations the diagonals are constant or logarithmic, and the off-diagonals have an inverse binomial form.

The matrix elements that reproduce - perfectly within fluctuations - the exact ones are

$$
H_{i i}=-\gamma_{1}^{\prime}\left(\ln \frac{i}{d}+1\right), \quad H_{i i+1}=\sigma^{\prime} \sqrt{N} \operatorname{nib}\left(\frac{i}{d}, N\right) .
$$

The subtraction in the ln term ensures that $H_{i i}$ is traceless. $\sigma^{\prime}$ and $\gamma_{1}^{\prime}$ are determined so as to yield $\sigma$ and $\gamma_{1}$ through

$$
\begin{aligned}
\sigma^{2} & =\left\langle\mathcal{H}^{2}\right\rangle=d^{-1} \sum H_{i i+1}^{2}+H_{i i-1}^{2}+H_{i i}^{2}, \\
\sigma^{3} \gamma_{1} & =\left\langle\mathcal{H}^{3}\right\rangle \simeq d^{-1} \sum H_{i i}\left(3 H_{i i+1}^{2}+3 H_{i i-1}^{2}+H_{i i}^{2}\right) .
\end{aligned}
$$

For the symmetric case $\left(\gamma_{1} \approx 0\right)$, the solution is simply $\sigma^{\prime} \approx \sigma$. For the asymmetric case, Eqs. (6) and 7 ) have to be solved. All parameters are given in the captions to Figs. 2 and 3

An approximation to the nib function is $\mathrm{Gauss}^{-1}=$ $x=\sqrt{-(2 N)^{-1} \ln g}$, the inverse of $g=\exp \left(-2 N x^{2}\right)$ obtained by expanding $b$ in Eq. (4) around $x=0$. Therefore $H_{i i+1}$ in Eq. (5) is approximated by

$$
H_{i+1}^{G}=\sqrt{-\left(\sigma^{\prime}\right)^{2} / 2 \ln (i / d)},
$$

which is seen in Figs. 2 and 3 to be fairly close to nib except for the severe overshooting at the origin that will become worse as $N$ increases. To fix ideas, think of the quotient of the direct functions: at $x=1 / 2$, $g(1 / 2) / b(1 / 2)=2^{N} e^{-N / 2} \approx e^{0.2 N}$.

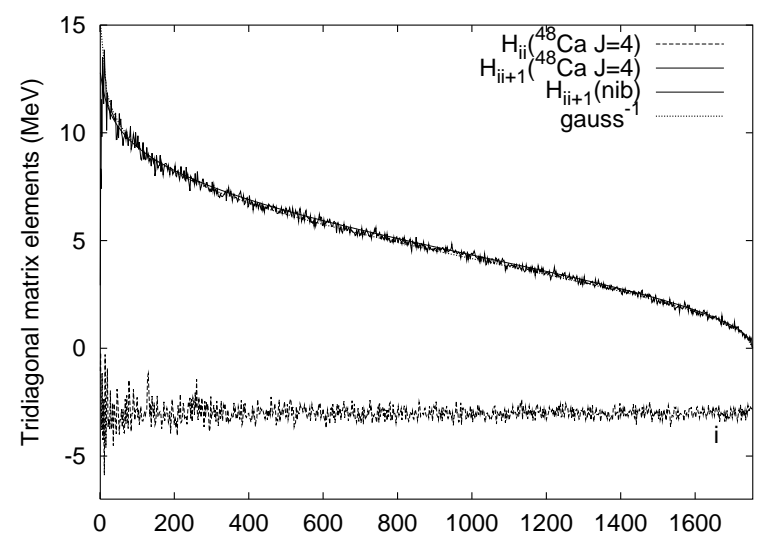

FIG. 2. Exact matrix elements for ${ }^{48} \mathrm{Ca}(J=4)$, the inverse binomial nib $\equiv H_{i i+1}$ (Eqs. (50) , and Gauss ${ }^{-1} \equiv H_{i i+1}^{G}$ (Eq. (8)). Parameters: $d=1755, N=10.78, \sigma=7.85 \mathrm{MeV}$, $\gamma_{1}=-.01$. See text

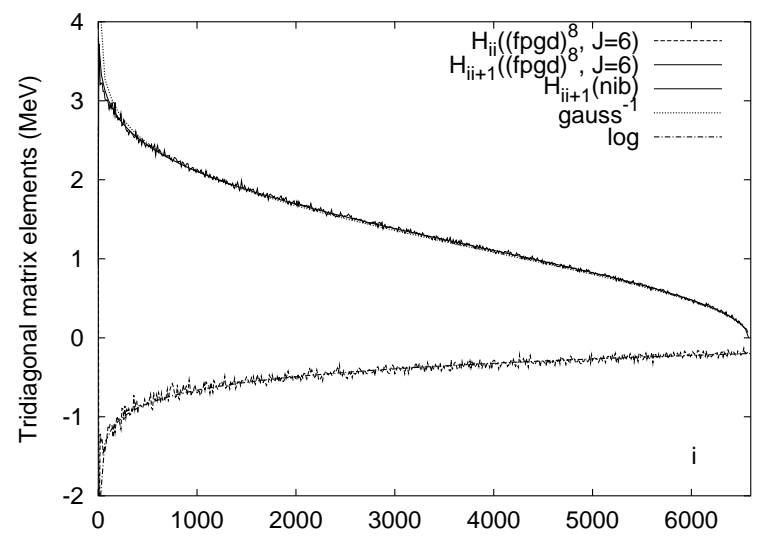

FIG. 3. Exact matrix elements (only one every ten are plotted) for $(f p g d)^{8}(J=6)$ compared with $\log \equiv H_{i i}$ (arbitrarily shifted), and nib $\equiv H_{i i+1}$ from Eqs. (5), and Gauss ${ }^{-1} \equiv H_{i i+1}^{G}$ (Eq. (8)) . Parameters: $d=6579, N=12.68, \sigma^{\prime}=2.14 \mathrm{MeV}$, $\sigma=2.18, \gamma_{1}^{\prime}=-0.246, \gamma_{1}=-0.296$. See text

Eqs. (5) are not fits but checked conjectures that should lead to the following CAT (Computer Assisted Theorem):

CAT. Hamiltonian matrices with no hidden symmetries have, except near the origin and within fluctuations, canonical Lanczos forms that depend only on their dimensionality, second and third moments and rank of the interaction.

No hidden symmetries imply that the matrices cannot be split in blocks. Which means that eigenvalues cannot be degenerate [3, page 300]. In other terms: the 
spectrum must have level repulsion. If this condition is not fulfilled, degeneracies (not detected by the Lanczos method), or semi-degeneracies (leading to strong fluctuations) will make it impossible to define a canonical form. Therefore, it is here that the condition of good quantum numbers comes in.

The rank dependence is crucial; e. g., for GOE matrices the equivalent of Eqs. (5) is $H_{i i}=0$ and $H_{i+1}=$ $\sqrt{1-i / d}$ (sic). (The cherry on the CAT cake)

The behaviour of the matrix at the origin depends on the pivot. The only general statement is that $H_{i+1}$ must be $(O \equiv$ of order $) O(\sigma)$ at the origin and raise to reach the nib line after $O(N)$ iterations. If the first diagonal is taken to be the lowest unperturbed state, or chosen variationally, it will be at an energy $O(N)$ below the $\log$ line and raise to reach it after $O(N)$ iterations. It seems a rather general fact that for such "good" pivots, diagonals and off-diagonals reach their respective lines in $\approx N / 2$ iterations [7,8]. It goes without saying that the correct descriptions of low-lying states and strength functions hinges on a good description at the origin. The contribution of Eqs. (5) is nonetheless essential: truncated calculations will have smaller $\sigma$ and the nib line will be lower. As a consequence, the ground state energy will not converge to the correct value and the density of states that determine the final aspect of the strength functions will be badly missed [8].

Eqs. (5) - eventually supplemented by the rapid raise at the origin - define an integrable problem, leading to a spectrum of locally equidistant levels (picket-fence) and locally uniform strength distributions. When fluctuations are switched on, the level spacings can be expected to follow a Wigner law, and the strengths a PorterThomas distribution [2]. To put it in other words: the system becomes chaotic and the strength is localized.

Eqs. (5), duly "dressed", amount to a general but constrained Anderson model, in which constants are replaced by $\log$ and nib functions, while behaviour at the origin and fluctuations (the dress) must be characterized in terms of properties of the Hamiltonian. It is likely that the large oscillations, $O(N)$ steps near the originas in Fig. 1 depend both on the pivot and on "details" of the Hamiltonian. Then, secular trends take over: fluctuations in the diagonals are about double that of the off-diagonals, and both seem to follow a random walk in which - at each step - it is more probable to keep the same direction than to reverse it.

Perfect dressing amounts to exact solutions, but model dressing is also possible and can be quite instructive.

For level densities, fluctuations matter less and it is obvious that the digonalization of the matrices defined by Eqs. (5) will yield densities very close to the exact ones. It is perhaps not so obvious that CAT has a corollary:

CAT corollary Level densities at fixed quantum numbers are binomial.
The checks are fully convincing for all the calculated cases, except when the dimensionalities become very small $(d \approx 100)$. Fig. 4 is an example.

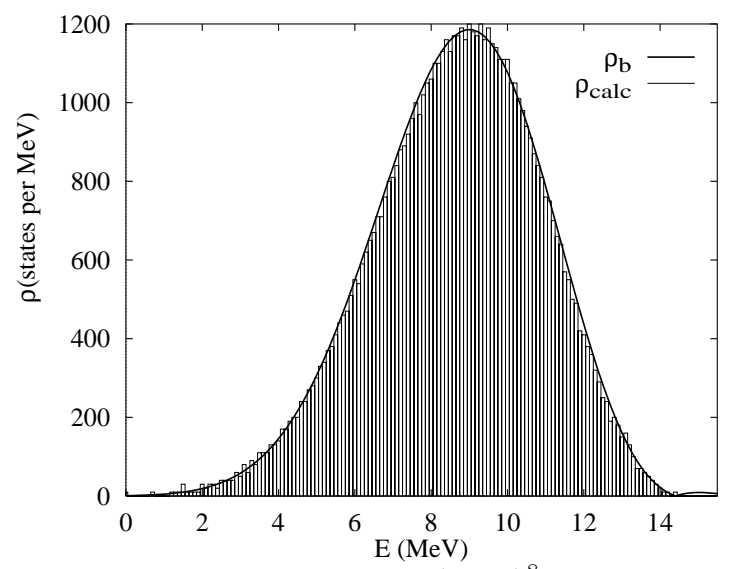

FIG. 4. Exact level densities for $(f p g d)^{8}, J=6$ compared with the binomial ones. Parameters from Fig. 3, treated as explained in [11, Eq. 5] $\left(d_{0}=1\right)$

The only significant problem is that the position of the ground state - which depends critically on the behaviour of the matrix at the origin - is not predicted with sufficient accuracy for spectroscopic purposes.

It should be noted that in Fig. A an Edgeworth corrected Gaussian [9] works as well as the binomial [10]. As explained after Eq. (8), binomials and Gaussiansand a fortiori Edgeworth corrected ones - can be close for the small $N$ accessible to simulations. But not in general.

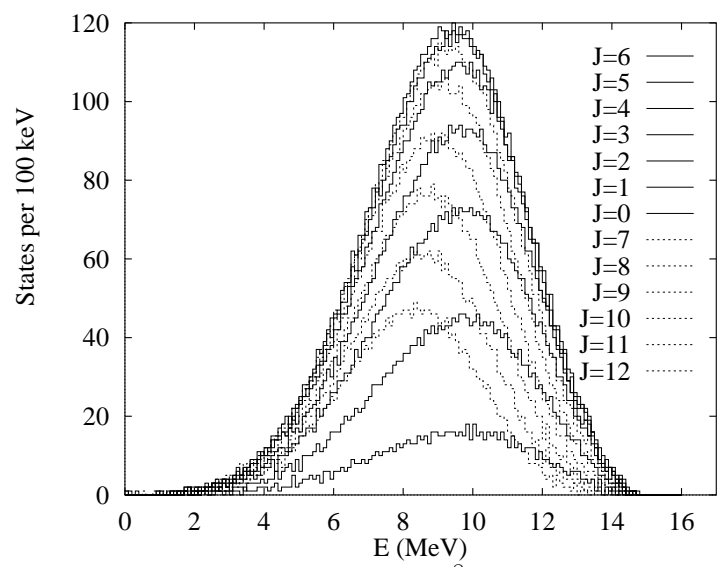

FIG. 5. Level densities for $(f p g d)^{8}, J=0,12$. The legend indicates the order in which $\rho_{J}$ appear as $d_{J}$ increases (full lines) or decreases (dashes)

For $(f p g d)^{8}$, the calculated multimodal total density, $\rho_{m}=d^{-1} \sum(2 J+1) \rho_{J}$, turns out to be as well described by a single binomial as the unimodal ones. The gentle buildup shown in Fig. 5 helps in understanding what happens. For ${ }^{48} \mathrm{Ca}$ the situation is the same. We have 
two examples of a multimodal $\rho_{m}$ behaving as unimodal, which squares with the systematic success of Bethe's formula [11]. Assuming that the signal for an eventual phase transitions is a significant deviation from binomial behaviour, the conclusion is that no low energy thermal phase transitions occur in nuclei.

For the individual $\rho_{J}$, Bethe's expression [12, Eq.(2B$62)$ ] amounts to $E_{c} \equiv J(J+1)$ and constant $\sigma^{2}$, whilefor $(f p g d)^{8}$-in Fig. 6, we find that both $E_{c}$ and $\sigma^{2}$ are nearly parabolic, the former with opposite sign to Bethe's. For ${ }^{48} \mathrm{Ca}, E_{c}$ is nearly constant and $\sigma^{2}$ again an inverted parabola. No reason for alarm, but the subject demands further study.

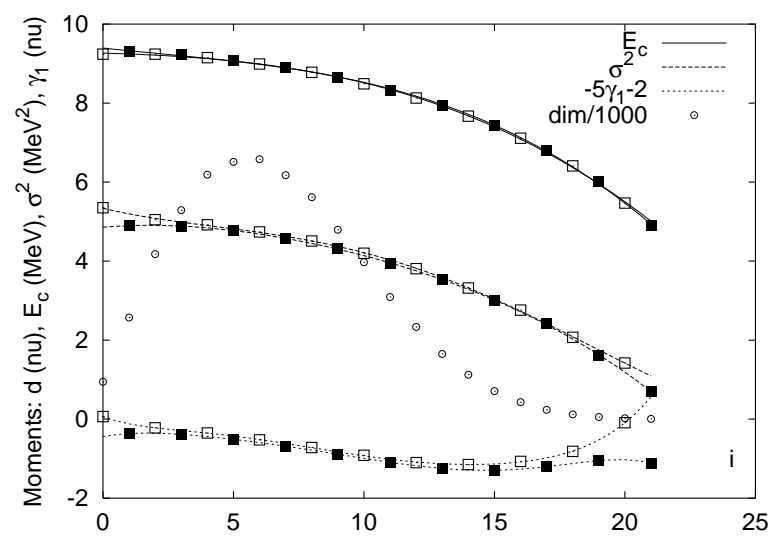

FIG. 6. Centroid $E_{c}, \sigma^{2},-5 \gamma_{1}-2$ and $d / 1000$ for $(f p g d)^{8}$. Full (open) squares for $J$ odd (even).

One thing is clear: we have to learn how to calculate low moments at fixed quantum numbers. A brute force approach is viable in very large spaces, but a bit of formalism could extend the range to arbitrarily large ones. The dimensionalities in Fig. 6 provide an example: they are the differences of two binomials, obtained by realizing that the calculation of the number of states at fixed $J_{z}$ is a single particle problem.

Once we have the moments, many things can be done. One of them is to treat random Hamiltonians and understand the canny insistence of $J=0$ to be ground state [13]. Probably the most promising is the calculation of thermodynamic quantities.

Thermodynamically, the behaviour of a single binomial is elementary. Write $\rho(n)=\lambda^{n}\left(\begin{array}{l}N \\ n\end{array}\right)$, with $E=n \epsilon$. Then $z(\beta)=\sum_{n} \rho(n) e^{-\beta \varepsilon n}=\left(1+\lambda e^{-\beta \varepsilon}\right)^{N}$, is the partition function and $E(\beta)=\partial \ln z / \partial \beta=N \varepsilon \lambda e^{-\beta \varepsilon} /\left(1+\lambda e^{-\beta \varepsilon}\right)$, the thermal energy: The same as obtained from a microcanonical calculation, $\beta=\partial \ln \rho / \partial E$, using the Stirling approximation for $\rho$. Therefore, continuous and discrete binomials have the same thermodynamics; The canonical partition function for a general problem is enormously simplified, as it becomes the sum over $x$, a set of conserved quantum numbers ( $E_{0 x}$ is the energy origin):

$$
Z(\beta)=\sum_{x}\left(1+\lambda_{x} e^{-\beta \varepsilon_{x}}\right)^{N_{x}} e^{\beta E_{0 x}},
$$

More on this soon [14].

Let us close by making explicit the striking complementarity between the approach in the previous paper, involving scalar traces $d^{-1}\left\langle\mathcal{H}^{K}\right\rangle$, and the Lanczos approach here, involving expectation values for a pivot at fixed quantum numbers, $\left\langle 0\left|\mathcal{H}^{K}\right| 0\right\rangle$. Low values of $K$ describe the centroid region, and are easy to find for the traces, while they describe the ground state in Lanczos and are hard to calculate. The converse is - or at least seems to be-true for high $K$. CAT is more general and has far more consequences than the CLT results [1], but they are theorems, while CAT is a conjecture. The theorems will be of help if they can be extended to traces at fixed quantum numbers. A task we have to tackle at any rate.

[1] Statistical theories of spectra: Fluctuations, C. E. Porter (Ed.), Academic Press, New York, 1965.

[2] T. A. Brody, J. Flores, J. B. French, P. A. Mello, A. Pandey end S. S. M. Wong, Rev. Mod. Phys. 53 (1981) 385.

[3] J. H. Wilkinson, The algebraic eigenvalue problem, OUP (1965).

[4] E. Caurier, A. Poves and A.P. Zuker, Phys. Rev. Lett. 74, 1517 (1995).

[5] E. Caurier, A.P. Zuker, A. Poves and G. Martínez Pinedo, Phys Rev C 50, 225 (1994).

[6] A. P. Zuker, J. Retamosa, A. Poves and E. Caurier, Phys. Rev. C 52, R1741 (1995).

[7] E. Caurier, G. Martínez, F. Nowacki, A. Poves, J. Retamosa and A. P. Zuker, INPC98, B. Frois et al. eds., Nuc. Phys. A 654 (1999) 747c

[8] L. Waha Ndeuma, Ph.D thesis, IReS 99-16, Strasbourg (1999).

[9] R. Haq, Can. J. Phys 68, 301 (1990).

[10] V. K. B. Kota, private communication.

[11] A. P. Zuker, previous paper.

[12] A. Bohr and B. Mottelson, Nuclear Structure vol I (Benjamin, Reading, 1964).

[13] C. W. Johnson, G. F. Bertsch, D. J. Dean and I. Talmi, Phys. Rev. C 61, 014311 (1999).

[14] G. Dussel and A. P. Zuker, in preparation. 